\title{
Outcomes of Single-Incision Thoracoscopic Surgery Using the Spinal Needle Anchoring Technique for Primary Spontaneous Pneumothorax
}

\author{
Seung Hyong Lee, M.D. ', Sun-Geun Lee, M.D. ${ }^{2}$, Sang-Ho Cho, M.D., Ph.D. ${ }^{2}$, Jae Won Song, M.D. ${ }^{2}$, \\ Dae Hyun Kim, M.D., Ph.D.2
}

'Department of Thoracic and Cardiovascular Surgery, Kyung Hee University Hospital, College of Medicine, Kyung Hee University; ${ }^{2}$ Department of Thoracic and Cardiovascular Surgery, Kyung Hee University Hospital at Gangdong, College of Medicine, Kyung Hee University, Seoul, Korea

\section{ARTICLE INFO}

Received November 1, 2021

Revised November 17, 2021

Accepted November 19, 2021

Corresponding author

Dae Hyun Kim

Tel $82-2-440-6158$

Fax 82-2-440-8004

E-mail kmctskdh@hanmail.net

ORCID

https://orcid.org/0000-0002-8434-7380

\begin{abstract}
Background: Although classical multi-port video-assisted thoracic surgery has been widely performed, single-incision thoracoscopic surgery (SITS) is a popular surgical technique for the treatment of primary spontaneous pneumothorax (PSP). However, the inconvenient alignment of instruments and the limited field of view occasionally make surgeons convert from SITS to multi-port surgery or extend the incision. This study aimed to present an easy and safe SITS technique for PSP using a spinal needle.

Methods: In total, 139 patients underwent SITS between May 2011 and December 2017. We used a spinal needle to hook the bulla or bleb, and wedge resection was performed through a small incision. Patients' medical records were reviewed retrospectively, and a telephone survey was conducted to investigate the recurrence rate.

Results: The mean age of the 139 patients was $23.62 \pm 9.60$ years. The mean operative time was 36.69 \pm 14.64 minutes, and multi-port conversion was not performed. The mean postoperative hospital stay was $3.00 \pm 0.78$ days, and the mean indwelling chest tube duration was $1.97 \pm 0.77$ days. No complications were observed. In the mean follow-up period of $86.75 \pm 23.20$ months, recurrence of pneumothorax was found in 3 patients.

Conclusion: We suggest that SITS for PSP with the aid of a spinal needle to replace a grasper is a safe and easy technique that only requires a small incision.
\end{abstract}

Keywords: Pneumothorax, Thoracoscopes, Video-assisted thoracic surgery

\section{Introduction}

Video-assisted thoracoscopic surgery (VATS) is widely used in the surgical treatment of spontaneous pneumothorax and is classically performed through several ports. Single-incision thoracoscopic surgery (SITS) for wedge pulmonary resection was first introduced by Rocco and colleagues [1], and numerous studies have reported that SITS provides less postoperative pain, lower paresthesia incidence, better cosmetic benefits, and a shorter recovery time than multiport VATS [2-5]. However, in classic SITS, the field of vision obtained with a single incision can at times be restricted because a video thoracoscope and 2 instruments (stapling instrument and grasping instrument) are simultaneously introduced through the same incision [6]. The risk of collision of instruments and the limited field of view can occasionally lead surgeons to convert from SITS to multi-port VATS or extend the incision line. This disadvantage can be overcome, at least in part, by meticulously using the instruments, by utilizing an articulated grasping instrument, and by reducing the size of the instruments. Surgeons have recently begun to investigate ways to reduce the number of instruments inserted through the incision site, and modified SITS techniques using percutaneous hook or anchoring suture have been developed that facilitate pulling of the target lesion without the need for making an incision at another site [6-9]. At our institution, we use a spinal needle that is $90 \mathrm{~mm}$ in length to create lung traction. Here, we report a single-institution experience by a single surgeon performing SITS for PSP using the method of spinal needle anchoring. 


\section{Methods}

\section{Patients}

In total, 139 patients with PSP were enrolled between May 2011 and December 2017 at Kyung Hee University Hospital at Gangdong. Approval from the institutional review board was obtained (approval no., KHNMC 2021-06012-002). Informed consent was obtained from all individual participants included in the study. Common indications for surgery were visible blebs or bullae found on chest computed tomography, air leakage for more than 3 days after closed thoracostomy, and recurrent ipsilateral pneumothorax.

\section{Surgical method}

Surgery was performed under general anesthesia, with the patient placed in the decubitus position, and the side was dependent on the side of pneumothorax. A $2.0-\mathrm{cm}$ skin incision was made in the first 10 patients and a $1.5-\mathrm{cm}$ skin incision in the 11th and subsequent patients at the mid-axillary line of the fifth intercostal space (Fig. 1A). If the patient already had a chest tube, an incision was made at the inserted site.

A 5-mm $30^{\circ}$ thoracoscope was inserted. The use of a wound retractor before insertion of the thoracoscope was optional. After insertion, an inspection of the lesion site using a grasping instrument for identifying a bulla/bullae or a bleb/blebs was performed. A $90^{\circ}$ bend at the end of the distal $2 \mathrm{~cm}$ of a $20 \mathrm{G}$ spinal needle was inserted through the anterior axillary line of the second intercostal space. The lesion was hooked to the spinal needle in the thoracic cavity using a grasping instrument. We found that the optimal spinal tap needle thickness was $20 \mathrm{G}$ because a needle under 19G was difficult to bend, and a needle over $21 \mathrm{G}$ may not overcome the lung parenchyma's weight. When hooking was completed and the resection angle was established, the thoracoscope was temporarily pulled out, and a straightened endoscopic stapler (Medtronic Endo-GIA; Medtronic, Minneapolis, MN, USA) and the thoracoscope were vertically inserted at the same time (Figs. 1B, 2). The endoscopic stapler was rotated and bent inside, and wedge resection was performed (Fig. 3). If the resection angle was

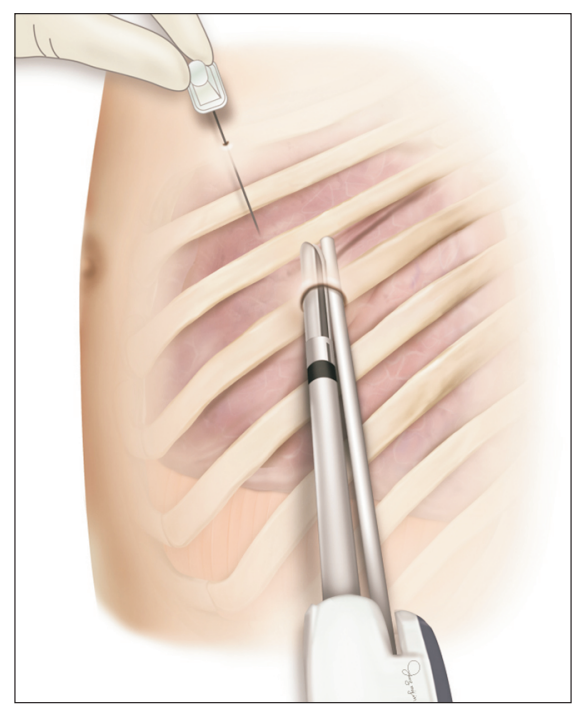

Fig. 2. Illustration of the operative field with all instruments when performing single-incision thoracoscopic surgery with the use of a spinal needle. The thoracoscope and stapling instrument are inserted through the same incision, and the needle is inserted in an independent location.
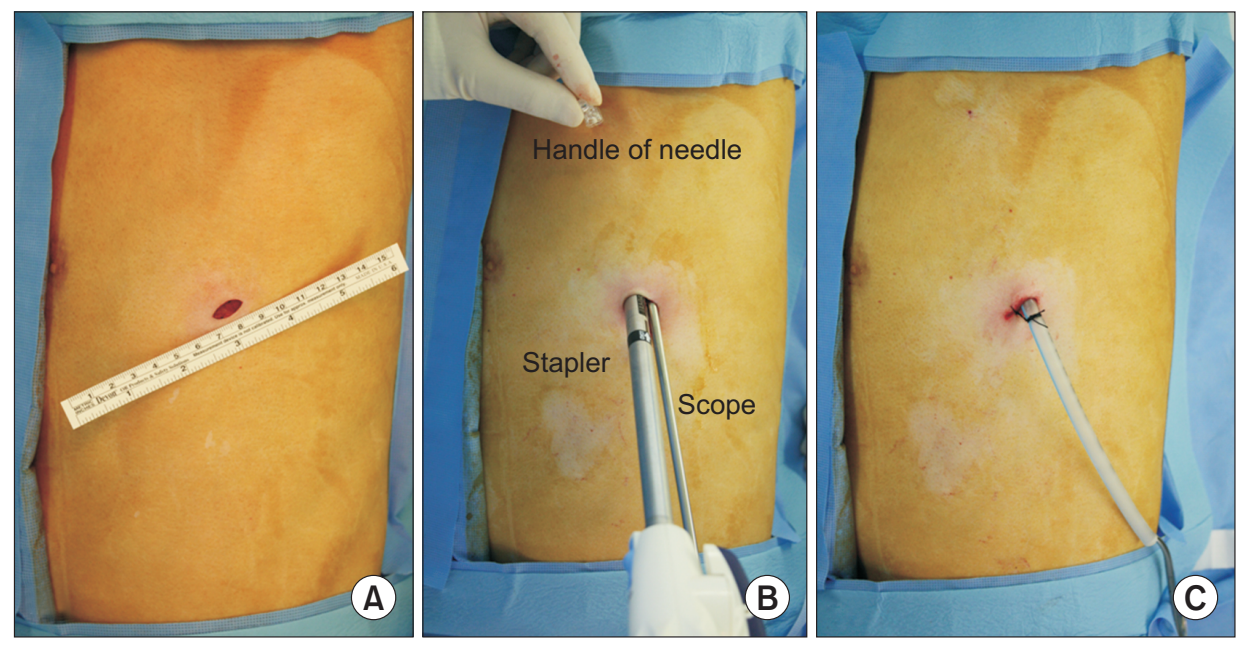

Fig. 1. Operative procedures of single-incision thoracoscopic surgery with the use of a spinal needle. (A) An approximately $1.5-\mathrm{cm}$ working window for surgery; (B) needle for hooking during surgery; and $(\mathrm{C})$ the postoperative wound. 


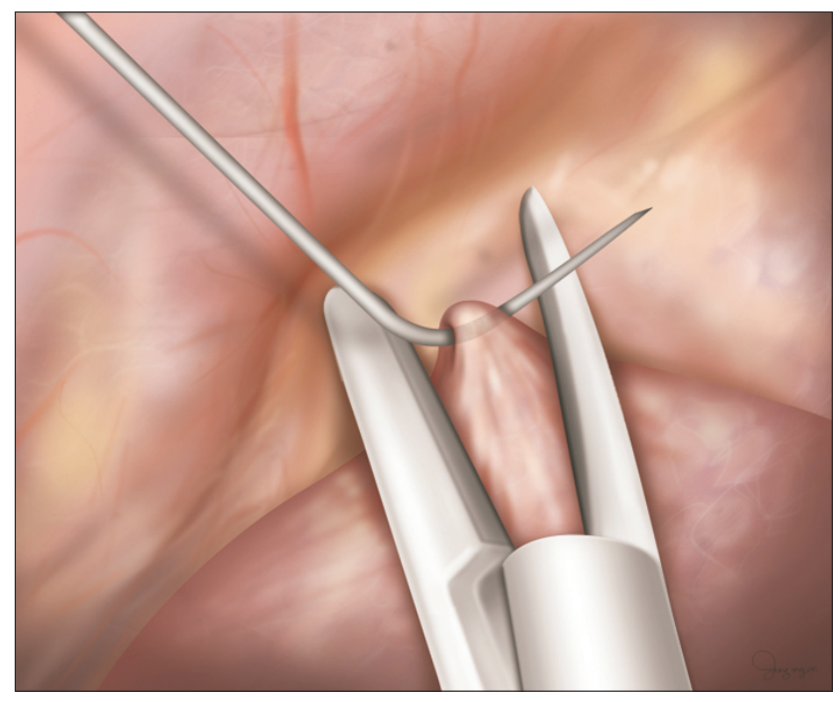

Fig. 3. Illustration before resection. The bleb is hooked by the spinal needle, and the endo stapler is properly positioned for wedge resection.

not efficient after the first stapling or if the target lesions were multifocal, we hooked another site to the same or another spinal needle and performed resection using an endoscopic stapler in the same manner.

When wedge resection was completed, an air leakage test was performed. Subsequently, the wedge resection site and surrounding lung parenchyma were covered with polyglycolic acid felt (Neoveil; Gunze Co. Ltd., Tokyo, Japan) and fibrin glue to reduce recurrence [10]. A 20F chest tube was placed at the incision site (Fig. 1C). A simple dressing was performed at the spinal needle insertion site.

The outcomes were evaluated, including operation time, duration of the postoperative hospital stay, indwelling chest tube duration, postoperative complications such as wound complications, prolonged air leak, bleeding including chest wall hematoma, and postoperative infection. Recurrence was evaluated using outpatient medical records. To identify postoperative recurrence accurately, we performed a telephone survey, in which we asked patients whether they had a history of recurrence at the same site of surgery.

\section{Results}

In total, 139 patients underwent SITS wedge resection at the Kyung Hee University Hospital in Gangdong. All operations were performed by a single surgeon at this center. Conversion to conventional VATS was not done.

Patients' mean age was $21.22 \pm 6.68$ years. The mean operative time was $36.69 \pm 14.64$ minutes, and the median
Table 1. Results of single-incision thoracoscopic surgery for primary spontaneous pneumothorax

\begin{tabular}{lc}
\hline \multicolumn{1}{c}{ Variable } & Value \\
\hline Total patients & 139 \\
Age $(\mathrm{yr})$ & $21.22 \pm 6.68$ \\
Operative time (min) & $36.69 \pm 14.64$ \\
Postoperative chest tube duration (day) & $1.97 \pm 0.77$ \\
Postoperative hospital stay (day) & $3.00 \pm 0.78$ \\
Postoperative complications & \\
$\quad$ Prolonged air leak & 0 \\
$\quad$ Bleeding & 0 \\
$\quad$ Wound infection & 0 \\
Recurrence & $3(2.16)$ \\
\hline
\end{tabular}

Values are presented as number, mean \pm standard deviation, or number (\%).

time was 30 minutes. The mean postoperative hospital stay was $3.00 \pm 0.78$ days, and the mean indwelling chest tube duration was $1.97 \pm 0.77$ days. Postoperative complications such as wound complications, prolonged air leak, bleeding, and infection were not observed. The mean follow-up period was $86.75 \pm 23.20$ months (range, 41-123 months), and recurrence of pneumothorax occurred in 3 patients $(2.16 \%)$ (Table 1).

\section{Discussion}

SITS is an advanced minimally invasive surgical procedure in which the surgeon operates exclusively through a single-entry point instead of the conventional 3 thoracoscopic incisions. Since SITS for wedge pulmonary resection was first introduced by Rocco et al. [1], the safety and feasibility of lung resections for PSP by SITS have been generally accepted based on a large number of studies [3-6,11]. Its morbidity is similar to that of multi-port VATS. The recurrence rate is comparable with the best results after multiport VATS or thoracotomy. Patients were satisfied with the small number of scars [4]. As reduced-port surgery, SITS offers clear advantages over multi-port VATS, particularly with regard to postoperative pain, chest wall paresthesia, and length of hospitalization [2,3,8,12-15]. However, SITS is not widely accepted by thoracic surgeons because of its disadvantages compared with conventional 3-port VATS. In classic SITS, the procedure was difficult to perform due to the presence of many instruments in the same incision site simultaneously, which unavoidably led to instrument fencing. Another disadvantage is that a relatively long skin incision can be required to minimize collisions between surgical instruments. In 2004, Rocco et al. [1] reported ef- 
ficacy for SITS requiring a skin incision of $2.5 \mathrm{~cm}$ in length. In the last decade, other investigators have reported classic SITS procedures with a $2.0-\mathrm{cm}$ incision [13]. To overcome these problems, a single-incision laparoscopic surgery port and an articulated grasping instrument were used when performing SITS [5,11]. Surgeons have recently begun to investigate ways to reduce the number of instruments inserted through the incision site, and modified SITS techniques have been developed that facilitate pulling of the target lesion using a percutaneous loop retractor, hook wire, or anchoring suture without the need for making an incision at another site [6-9].

Chong et al. [7] reported the "tower crane technique" with a 15-mm trocar using anchoring sutures to the lung. Mogi et al. [8] described the SITS technique using a thin puncture device (Mini Loop Retractor). Son et al. [6] reported acceptable clinical outcomes in 104 patients who underwent SITS using the anchoring suture technique. Unlike these anchoring techniques, we used a spinal needle that was $90 \mathrm{~mm}$ in length to hook the lesion and create lung traction. It did not share the working window with the other instruments. This feature makes the procedure functionally similar to 2-incision thoracoscopic surgery. In addition, a spinal needle does not require another incision or a surgical wound. Even if repeated insertions occur, the operation can be considered as SITS. With these advantages, this technique requires only a $1.5-\mathrm{cm}$ incision. Our center started using this technique in 2011 with a $2.0-\mathrm{cm}$ incision. From 2012, we succeeded in reducing the incision to $1.5 \mathrm{~cm}$ with experiential improvements. We believe that this incision length is the shortest among the heretofore reported SITS techniques for the treatment of pneumothorax. Another advantage of this strategy is that it is easy to change the hooking site of the lung, unlike other methods, because hooking the target lesion and removing the spinal needle from the lesion can be easily performed in the thoracic cavity using a grasping instrument. Manipulation of the hooked target lesion can also be performed easily.

In our present study, the mean operative time was $36.69 \pm 14.64$ minutes, and the mean postoperative hospital stay was $3.00 \pm 0.78$ days. Postoperative complications were not observed, and recurrence of pneumothorax occurred in 3 patients $(2.16 \%)$. Although we did not establish a multi-port VATS group or a classic SITS group for comparison, our data showed acceptable results, comparable with those of other studies.

It takes a long time to become an expert in SITS, and the learning curve requires 30 to 100 cases [16-18]. However, this technique makes SITS more accessible and serves as a bridge to perform SITS without a needle (e.g., lobectomy) in the future.

Some aspects of this technique need to be improved, such as the weak anchoring power compared to the grasping instrument, the difficulty in releasing the hand from the spinal needle because of excessive rotational potential, and the difficulty in maneuvering the spinal needle into the thoracic cavity of some patients (e.g., those who are obese). In obese patients, we overcame this problem by using a $19 \mathrm{G}$ needle and bending at $3 \mathrm{~cm}$ on the distal end rather than $2 \mathrm{~cm}$.

In conclusion, SITS for PSP with the aid of a spinal needle to replace the grasper is a safe and easy technique that can be performed with a minimal incision.

\section{Conflict of interest}

No potential conflict of interest relevant to this article was reported.

\section{ORCID}

Seung Hyong Lee: https://orcid.org/0000-0002-9839-6431

Sun-Geun Lee: https://orcid.org/0000-0002-7509-0229

Sang-Ho Cho: https://orcid.org/0000-0001-5590-1904

Jae Won Song: https://orcid.org/0000-0002-3530-0623

Dae Hyun Kim: https://orcid.org/0000-0002-8434-7380

\section{References}

1. Rocco G, Martin-Ucar A, Passera E. Uniportal VATS wedge pulmonary resections. Ann Thorac Surg 2004;77:726-8.

2. Jutley RS, Khalil MW, Rocco G. Uniportal vs standard three-port VATS technique for spontaneous pneumothorax: comparison of post-operative pain and residual paraesthesia. Eur J Cardiothorac Surg 2005;28:43-6.

3. Yang Y, Dong J, Huang Y. Single-incision versus conventional threeport video-assisted surgery in the treatment of pneumothorax: a systematic review and meta-analysis. Interact Cardiovasc Thorac Surg 2016;23:722-8.

4. Masmoudi H, Etienne H, Sylvestre R, et al. Three hundred fifty-one patients with pneumothorax undergoing uniportal (single port) video-assisted thoracic surgery. Ann Thorac Surg 2017;104:254-60.

5. Yang HC, Cho S, Jheon S. Single-incision thoracoscopic surgery for primary spontaneous pneumothorax using the SILS port compared with conventional three-port surgery. Surg Endosc 2013;27:139-45.

6. Son BS, Kim DH, Lee SK, Kim CW. Small single-incision thoracoscopic surgery using an anchoring suture in patients with primary spontaneous pneumothorax: a safe and feasible procedure. Ann Tho- 
rac Surg 2015;100:1224-9.

7. Chong Y, Cho HJ, Kang SK, et al. Outcomes of the tower crane technique with a $15-\mathrm{mm}$ trocar in primary spontaneous pneumothorax. Korean J Thorac Cardiovasc Surg 2016;49:80-4.

8. Mogi A, Yamaki E, Kosaka T, Asao T, Kuwano H. Thoracoscopic wedge resection through a single incision using a thin puncture device. Ann Thorac Cardiovasc Surg 2014;20:198-201.

9. Ng CS, Hui JW, Wong RH. Minimizing single-port access in video-assisted wedge resection, with a hookwire. Asian Cardiovasc Thorac Ann 2013;21:114-5.

10. Hong KP, Kim DK, Kang KH. Staple line coverage with a polyglycolic acid patch and fibrin glue without pleural abrasion after thoracoscopic bullectomy for primary spontaneous pneumothorax. Korean J Thorac Cardiovasc Surg 2016;49:85-91.

11. Berlanga LA, Gigirey O. Uniportal video-assisted thoracic surgery for primary spontaneous pneumothorax using a single-incision laparoscopic surgery port: a feasible and safe procedure. Surg Endosc 2011;25:2044-7.

12. Jung H, Oh TH, Cho JY, Lee DH. Mid-term outcomes of single-port versus conventional three-port video-assisted thoracoscopic surgery for primary spontaneous pneumothorax. Korean J Thorac Cardiovasc Surg 2017;50:184-9.
13. Kang DK, Min HK, Jun HJ, Hwang YH, Kang MK. Early outcomes of single-port video-assisted thoracic surgery for primary spontaneous pneumothorax. Korean J Thorac Cardiovasc Surg 2014;47: 384-8.

14. Kapicibasi HO. Uniportal VATS technique for primary spontaneous pneumothorax: an analysis of 46 cases. Pak J Med Sci 2020;36:2248 .

15. Tamura M, Shimizu Y, Hashizume Y. Pain following thoracoscopic surgery: retrospective analysis between single-incision and three-port video-assisted thoracoscopic surgery. J Cardiothorac Surg 2013;8: 153.

16. Drevet G, Ugalde Figueroa P. Uniportal video-assisted thoracoscopic surgery: safety, efficacy and learning curve during the first 250 cases in Quebec, Canada. Ann Cardiothorac Surg 2016;5:100-6.

17. Martin-Ucar AE, Aragon J, Bolufer Nadal S, et al. The influence of prior multiport experience on the learning curve for single-port thoracoscopic lobectomy: a multicentre comparative study. Eur J Cardiothorac Surg 2017;51:1183-7.

18. Yang HC, Kim S, Yum S, Cho S, Kim K, Jheon S. Learning curve of single-incision thoracoscopic surgery for primary spontaneous pneumothorax. Surg Endosc 2017;31:1680-7. 